\title{
The phase transition phenomena in anisotropic superconductors : effect of the orthorhombic crystal field and the potential impurity scattering.
}

\author{
Grzegorz Harańt, Jason Taylor and A. D. S. Nagi \\ Department of Physics, University of Waterloo, Waterloo, Ontario, Canada, N2L 3G1
}

(December 5, 1996)

\begin{abstract}
A combined effect of the orthorhombic crystal field and potential impurity scattering on several superconducting states of a tetragonal symmetry is studied within a weak-coupling mean field approach. It is shown that the nonmagnetic impurities stabilize the states belonging to the identity irreducible representation. The electronic specific heat jump at the phase transition is analyzed. Its dependence on the potential scattering rate for large impurity concentration is shown to be remarkably different for the states with a nonzero value of the Fermi surface averaged order parameter than for those with a vanishing one. In particular, very distinct signals from $d_{x^{2}-y^{2}}$ state in YBCO and $d_{x y}$ state in BSCCO compound are predicted. This effect may be used as a test for the presence of these states in the above cuprates.
\end{abstract}

Keywords: d-wave superconductor, Fermi surface, pair breaking, specific heat at $T_{c}$, weak coupling 


\section{INTRODUCTION}

It is generally agreed that a key structural element of the high- $T_{c}$ superconductors is the quasi-two dimensional copper-oxygen plane. In most of the cuprates this plane has nearly a tetragonal symmetry with a small orthorhombic distortion. 1 The unit cells of some of these materials contain other elements, like the one-dimensional copper-oxygen chains in $\mathrm{YBa}_{2} \mathrm{Cu}_{3} \mathrm{O}_{7-\delta}(\mathrm{YBCO})$ which reduce their symmetry to the orthorhombic one $\left(C_{2 v}\right)$. It is possible that the $\mathrm{Cu}-\mathrm{O}$ chains may also contribute to the superconducting behavior of YBCO as the in-plane transport properties display an appreciable anisotropy significantly larger than that expected from a slight orthorhombicity of the $\mathrm{CuO}_{2}$ planes. Several experiments2 2 were designed to measure the transport properties separately for the $a$ and $b$ directions of the copper-oxygen planes. In YBCO, a large anisotropy of about $50 \%$ between the $a$ and $b$ directions has been observed $\mathrm{A}$. $\mathrm{s}$ in the zero temperature penetration depth $\left(\lambda_{a}(0) / \lambda_{b}(0) \approx 1.55\right)$ and a smaller anisotropy has been seen in the thermal conductivity (maximum value of $\kappa_{a} / \kappa_{b} \approx 1.15$ occurring near $40 \mathrm{~K}$ ). . 1 . Also the microwave absorption measurements $\mathrm{B}$. $\mathrm{G}$ in YBCO show a significant in-plane anisotropy in the surface resistance and in the infrared conductivity, the later remaining about a factor of 2 larger in the $b$ direction for the temperature range of 10-90K. The in-plane anisotropy of the surface resistance 3 in the normal state of YBCO agrees with the observed dc resistivity anisotropy 7 目 $\varrho_{a} / \varrho_{b} \sim 2$, which was indicated by the band-structure calculations 10 as well. Although very weak orthorhombicity due to a superlattice distortion in the Bi-O layer, $\mathrm{Bi}_{2} \mathrm{Sr}_{2} \mathrm{CaCu}_{2} \mathrm{O}_{8}$ (BSCCO) displays $a b$-plane anisotropy 9 of about $10 \%$ in the optical conductivity and the dc resistivity. It has been pointed out,11, 2 that in an orthorhombic system the superconducting order parameter becomes a mixture of $d$-wave and $s$-wave components as they belong to the same irreducible representation of the crystal point group. This leads to an interesting feature in the specific heat jump at the phase transition in the presence of a potential impurity scattering. 13

Using a simplified single-band model simulating the orthorhombic anisotropy of the Fermi 
surface for YBCO and BSCCO, we have analyzed a group of superconducting states in the untwinned systems. We have studied the effect of potential scattering on the stability of these states and also on the jump in the electronic specific heat at the phase transition.

\section{ORDER PARAMETER AND FERMI SURFACE ANISOTROPY}

We consider a singlet superconducting order parameter with its orbital part defined as follows

$$
\Delta(\mathbf{k})=\Delta e(\mathbf{k})
$$

where $e(\mathbf{k})$ is a basis real function of a one-dimensional (1D) irreducible representation of $C_{4 v}$ point group. We normalize $e(\mathbf{k})$ by taking its average value over the Fermi surface (FS) $\left\langle e^{2}\right\rangle=\int_{F S} d S_{k} n(\mathbf{k}) e^{2}(\mathbf{k})=1$, where $\int_{F S} d S_{k}$ represents the integration over the Fermi surface and $n(\mathbf{k})$ is the angle resolved FS density of states, which obeys $\int_{F S} d S_{k} n(\mathbf{k})=1$. This normalization gives $\Delta$ the meaning of the absolute magnitude of the order parameter. Our discussion is limited to the functions $e(\mathbf{k})$ confined to the $\mathrm{XY}$ plane only, which seems to be appropriate for the high $T_{c}$ compounds.22 These functions are listed in Tab. 1, with the symmetry group notation after Ref. 14 . The basis functions of the $C_{4 v}$ irreducible representations $\Gamma_{1}^{+}, \Gamma_{3}^{+}$and $\Gamma_{4}^{+}$are taken as three linearly independent second order polynomials. A fourth order polynomial is used then as a basis function of the remaining $\Gamma_{2}^{+}$representation. Finally, for the sake of comparison, we consider two more fourth order polynomials belonging to the identity irreducible representation $\Gamma_{1}^{+}$. The superconducting states given by the functions $e(\mathbf{k})$ constructed from the angular momentum eigenfunctions corresponding to a quantum number $\mathrm{L}=2$ are called the $d$-wave states (second order polynomials) and those for which the $\mathrm{L}=4$ eigenfunctions were used are called the $g$-wave states (fourth order polynomials). Unless an ambiguity arises we will use a more informal name of extended s-wave for the states which are invariant under rotation through $\pi / 2$ about the $z$ axis, that is for $\Gamma_{1}^{+}$ and $\Gamma_{2}^{+}$of $C_{4 v}$ irreducible representations given in Tab. 1. 
The presence of the orthorhombic anisotropy means that the relevant point group is a subgroup of the square, which is not the same for all the cuprate superconductors.12 In the case of $\mathrm{YBCO}$, the $a$ - and $b$ - crystal axes become inequivalent while in BSCCO the two orthogonal $45^{\circ}$ axes do so. Therefore the group classification of the superconducting states differs in YBCO and BSCCO compounds (see Tab. 1). A rotation of a coordinate system through $45^{\circ}$ about the z-axis transforms a YBCO-type geometry into a BSCCO-type one and also shows an equivalence of the superconducting states, which we summarize in Tab. 2. Distinguishing between these two symmetries, we make a simple approximation of the orthorhombic anisotropy by assuming the following form of the electron band国, 国 energy $\xi_{\mathbf{k}}$ measured from the Fermi energy $\varepsilon_{F}$ level

$$
\begin{gathered}
\xi_{\mathbf{k}}=c_{x} k_{x}^{2}+c_{y} k_{y}^{2}-\varepsilon_{F}, \quad \text { for } \mathrm{YBCO} \\
\xi_{\mathbf{k}}=\frac{1}{2}\left(c_{x}+c_{y}\right)\left(k_{x}^{2}+k_{y}^{2}\right)+\left(c_{x}-c_{y}\right) k_{x} k_{y}-\varepsilon_{F}, \quad \text { for } \mathrm{BSCCO}
\end{gathered}
$$

A dimensionless ratio of the effective masses $c_{x} / c_{y}$ becomes a parameter describing the orthorhombic anisotropy of the Fermi surface. It changes from 0 to 1 , with $c_{x} / c_{y}=1$ describing a circular FS and $c_{x} / c_{y}=0$ corresponding to a one-dimensional limit, non physical for the cuprates. For the sake of simplicity the orthorhombic symmetry is introduced as a deviation from a cylindrical FS not from a tetragonal one. We assume that this simplified single-band mode 11 given by Eqs. (2) and (3) captures some essential anisotropic features of both types of orthorhombicity i.e. that of YBCO and BSCCO. Particularly in the case of YBCO, where the symmetry lowering is mainly due to the existence of the chains, limiting the superconducting electrons to the $\mathrm{CuO}_{2}$ planes only and introducing the crystal field anisotropy by an elliptical Fermi surface is to be understood as a first approximation which reflects the basic properties related to the orthorhombicity of the compound. We employ the above singleband model to study the effect of orthorhombic anisotropy on the critical temperature $T_{c}$ and the specific heat jump at the phase transition in the presence of nonmagnetic impurity scattering. 


\section{NONMAGNETIC IMPURITY SCATTERING NEAR $\mathbf{T}_{\mathbf{C}}$}

The single-particle Green's function in the presence of nonmagnetic, noninteracting impurities is expressed in Nambu space as

$$
\hat{G}(\omega, \mathbf{k})=-\frac{1}{\tilde{\omega}^{2}+\xi_{k}^{2}+|\tilde{\Delta}(\mathbf{k})|^{2}}\left(i \tilde{\omega} \hat{\tau}_{0}+\xi_{k} \hat{\tau}_{3}+\tilde{\Delta}(\mathbf{k}) \hat{\tau}_{2}\right)
$$

where $\hat{\tau}_{0}$ and $\hat{\tau}_{i}(i=1,2,3)$ represent the unit and Pauli matrices in particle-hole space respectively and $\xi_{k}$ is the quasiparticle energy (Eqs. (2), (3)). The renormalized Matsubara frequency $\tilde{\omega}(\mathbf{k})$ and the renormalized order parameter $\tilde{\Delta}(\mathbf{k})$ are given by

$$
\tilde{\omega}=\omega-\Sigma_{0}, \quad \tilde{\Delta}(\mathbf{k})=\Delta(\mathbf{k})+\Sigma_{1}
$$

with $\omega=\pi T(2 n+1)$ ( $\mathrm{T}$ is the temperature, $\mathrm{n}$ is an integer). $\Sigma_{0}, \Sigma_{1}$ are the self-energies due to the electron-impurity scattering obtained in the t-matrix approximation. 16.17 This approach introduces two parameters describing the scattering process: $c=1 /\left(\pi N_{0} V_{i}\right)$ and $\Gamma=n_{i} / \pi N_{0}$, where $N_{0}, V_{i}$ and $n_{i}$ are respectively the overall density of states at the Fermi surface (FS), the impurity (defect) potential and the impurity concentration. We assume $s$-wave scattering by the impurities, that is, $V_{i}$ does not have an internal momentumdependence.18 It is particularly convenient to think of $c$ as a measure of the scattering strength, with $c=0$ in the unitary limit and $c \gg 1$ for weak scattering that is the Born limit. Assuming a particle-hole symmetry of the quasiparticle spectrum we get the selfenergies defined as follows

$$
\Sigma_{0}=-\Gamma \frac{g_{0}}{c^{2}+g_{0}^{2}+g_{1}^{2}}, \quad \Sigma_{1}=\Gamma \frac{g_{1}}{c^{2}+g_{0}^{2}+g_{1}^{2}}
$$

with $g_{0}, g_{1}$ functions determined by the self-consistent equations

$$
\begin{aligned}
& g_{0}=\frac{1}{N_{0} \pi} \sum_{\mathbf{k}} \frac{\tilde{\omega}}{\tilde{\omega}^{2}+\xi_{k}^{2}+|\tilde{\Delta}(\mathbf{k})|^{2}} \\
& g_{1}=\frac{1}{N_{0} \pi} \sum_{\mathbf{k}} \frac{\tilde{\Delta}(\mathbf{k})}{\tilde{\omega}^{2}+\xi_{k}^{2}+|\tilde{\Delta}(\mathbf{k})|^{2}}
\end{aligned}
$$


The self-consistency equation for the order parameter reads

$$
\Delta(\mathbf{k})=-T \sum_{\omega} \sum_{\mathbf{k}^{\prime}} V\left(\mathbf{k}, \mathbf{k}^{\prime}\right) \frac{\tilde{\Delta}\left(\mathbf{k}^{\prime}\right)}{\tilde{\omega}^{2}+\xi_{k^{\prime}}^{2}+\left|\tilde{\Delta}\left(\mathbf{k}^{\prime}\right)\right|^{2}}
$$

where $V\left(\mathbf{k}, \mathbf{k}^{\prime}\right)$ is the phenomenological pair potential taken as

$$
V\left(\mathbf{k}, \mathbf{k}^{\prime}\right)=-V_{0} e(\mathbf{k}) e\left(\mathbf{k}^{\prime}\right)
$$

To proceed further, we restrict the wave vectors of the electron self-energy and pairing potential to the Fermi surface and replace $\sum_{\mathbf{k}}$ by $N_{0} \int_{F S} d S_{k} n(\mathbf{k}) \int d \xi_{k}$. Integrating over $\xi_{k}$, the gap equation (9) can be transformed after a standard procedure 19 into

$$
\ln \left(\frac{T}{T_{c_{0}}}\right)=2 \pi T \sum_{\omega \geq 0}\left(\int_{F S} d S_{k} n(\mathbf{k}) f(\omega, \mathbf{k})-\frac{1}{\omega}\right)
$$

where the $f(\omega, \mathbf{k})$ function is defined as follows

$$
f(\omega, \mathbf{k})=\frac{e(\mathbf{k}) \tilde{\Delta}(\mathbf{k})}{\Delta\left[\tilde{\omega}^{2}+|\tilde{\Delta}(\mathbf{k})|^{2}\right]^{\frac{1}{2}}}
$$

and $T_{c_{0}}$ is the critical temperature in the absence of impurities. We expand the gap equation ([1]) in powers of $\Delta^{2}$ around $\Delta=0$ taking into account that $\tilde{\omega}$ and $\tilde{\Delta}$ are functions of $\Delta^{2}$ as given by Eqs. (5)-(\$). Keeping up to the second power terms in $\Delta$ we get the GinzburgLandau approximation of the gap equation

$$
\ln \left(\frac{T}{T_{c_{0}}}\right)=-f_{0}-\frac{1}{2} f_{1}\left(\frac{\Delta}{2 \pi T}\right)^{2}
$$

where the coefficients are given by

$$
\begin{gathered}
f_{0}=-2 \pi T \sum_{\omega>0}\left(\int_{F S} d S_{k} n(\mathbf{k})(f(\omega, \mathbf{k}))_{\Delta=0}-\frac{1}{\omega}\right) \\
f_{1}=-(2 \pi T)^{3} \sum_{\omega} \int_{F S} d S_{k} n(\mathbf{k})\left(\frac{d f(\omega, \mathbf{k})}{d \Delta^{2}}\right)_{\Delta=0}
\end{gathered}
$$

Taking the derivatives with respect to $\Delta^{2}$

$$
\frac{d}{d \Delta^{2}}=\frac{\partial}{\partial \Delta^{2}}+\sum_{\omega}\left\{\frac{d \tilde{\omega}}{d \Delta^{2}} \frac{\partial}{\partial \tilde{\omega}}+\frac{d \tilde{\Delta}(\mathbf{k})}{d \Delta^{2}} \frac{\partial}{\partial \tilde{\Delta}(\mathbf{k})}\right\}
$$


and with a use of the relations given in Eqs. (5)-(8) and (12) we obtain

$$
\begin{aligned}
& f_{0}=\left(1-\langle e\rangle^{2}\right)\left(\psi\left(\frac{1}{2}+\varrho\right)-\psi\left(\frac{1}{2}\right)\right) \\
& f_{1}=2\langle e\rangle\left[2\left\langle e^{3}\right\rangle+5\langle e\rangle^{3}-7\langle e\rangle\right] \varrho^{-2}\left(\psi\left(\frac{1}{2}+\varrho\right)-\psi\left(\frac{1}{2}\right)\right) \\
& +2\langle e\rangle\left[-2\left\langle e^{3}\right\rangle-3\langle e\rangle^{3}+5\langle e\rangle\right] \varrho^{-1} \psi^{(1)}\left(\frac{1}{2}+\varrho\right)+4\langle e\rangle^{2}\left[1-\langle e\rangle^{2}\right] \varrho^{-1} \psi^{(1)}\left(\frac{1}{2}\right) \\
& +\frac{1}{2}\left[-\left\langle e^{4}\right\rangle+3\langle e\rangle^{4}+4\langle e\rangle\left\langle e^{3}\right\rangle-6\langle e\rangle^{2}\right] \psi^{(2)}\left(\frac{1}{2}+\varrho\right)-\frac{1}{2}\langle e\rangle^{4} \psi^{(2)}\left(\frac{1}{2}\right) \\
& +\frac{1}{6}\left[2\left(\langle e\rangle^{2}-1\right)^{2} \frac{1}{c^{2}+1}-\langle e\rangle^{4}+2\langle e\rangle^{2}-1\right] \varrho \psi^{(3)}\left(\frac{1}{2}+\varrho\right)
\end{aligned}
$$

where $\varrho=\Gamma /\left[\left(c^{2}+1\right) 2 \pi T\right]$ and $\psi, \psi^{(n)}(n=1,2,3)$ are the polygamma functions.20 In the unitary limit $c=0$ and $\varrho=\Gamma /(2 \pi T)$. Alternatively for weak scattering $(c \gg 1)$ we obtain the Born scattering rate $\varrho=\pi N_{0} n_{i} V_{i}^{2} /(2 \pi T)$ and also neglect $\varrho /\left(c^{2}+1\right)$ in the last term of Eq. (18). Coefficients $f_{0}$ and $f_{1}$ involve three different types of the Fermi surface averages of the superconducting order parameter namely, $\langle e\rangle,\left\langle e^{3}\right\rangle$, and $\left\langle e^{4}\right\rangle$, which depend on the orthorhombic anisotropy parameter $c_{x} / c_{y}$.21 These averages enter the free energy and determine the thermodynamic properties at the phase transition. While the critical temperature $T_{c}$ is determined by the $f_{0}$ function and therefore is characterized only by $\langle e\rangle$, the other thermodynamic quantities like the entropy or the specific heat for instance, involve, through the $f_{1}$ function (Eq. (18)), the Fermi surface average values of higher powers of $e(\mathbf{k})$. It is illustrative for the purpose of this paper to show these averages as functions of the orthorhombic anisotropy parameter $c_{x} / c_{y}$. We present $\langle e\rangle,\left\langle e^{3}\right\rangle$, and $\left\langle e^{4}\right\rangle$ in Figs. 1a - 1c respectively, where the curve numbers correspond to the states listed in Tab. 2. The analytical expressions of these averages for the YBCO symmetry (Eq. (2)) are given in the Appendix. It is worth mentioning here, that as the $k_{x}^{2}-k_{y}^{2}$ state $\left(d_{x^{2}-y^{2}}\right)$ belongs to different irreducible representations (Tab. 1) in YBCO $\left(\Gamma_{1}^{+}\right)$and BSCCO $\left(\Gamma_{3}^{+}\right)$, its FS average value 
$\langle e\rangle$ is non zero in the first compound $\left(c_{x} / c_{y}<1\right)$, but $\langle e\rangle$ is unchanged by the orthorhombic crystal field and is zero in the second one. Further, for the $k_{x} k_{y}$ state $\left(d_{x y}\right)\langle e\rangle=0$ in YBCO $\left(\Gamma_{3}^{+}\right)$and $\langle e\rangle \neq 0$ in BSCCO $\left(\Gamma_{1}^{+}\right)$. These facts are of crucial importance for the critical temperature and the jump in the electronic specific heat at the phase transition, which we discuss in the following sections.

\section{CRITICAL TEMPERATURE $\mathbf{T}_{\mathbf{C}}$}

We analyze in the Ginzburg-Landau (G-L) regime the stability of different superconducting states in the presence of impurity potential scattering and the orthorhombic crystal field. For any FS we consider the states of the same critical temperature in the absence of impurities $\left(T_{c_{0}}\right)$, which means that we normalize the order parameter eigenfunctions as $\left\langle e^{2}\right\rangle=1.22$ Then we look at the impurity effect on $T_{c}$ of these states. It should be noted that since the orthorhombic anisotropy changes the density of states at the Fermi level, $T_{c_{0}}$ is different for different values of $c_{x} / c_{y}$ even with the condition $\left\langle e^{2}\right\rangle=1$. We are interested in the influence of impurity scattering on the states of different symmetry but with the same $T_{c_{0}}$ in the presence of a given orthorhombic crystal field i.e. for a given $c_{x} / c_{y}$ value. The G-L free energy difference $\Delta F=F_{s}-F_{n}$ between the superconducting $\left(F_{s}\right)$ and the normal $\left(F_{n}\right)$ phase written up to the fourth order terms in $\Delta$

$$
\Delta F=\alpha \Delta^{2}+\frac{1}{2} \beta \Delta^{4}
$$

with the coefficients $\alpha$ and $\beta$ determined from Eqs. (13), (17) and (18) i.e.

$$
\begin{gathered}
\alpha=N_{0}\left(\ln \left(\frac{T}{T_{c_{0}}}\right)+f_{0}\right) \\
\beta=\frac{N_{0} f_{1}}{2\left(2 \pi T_{c}\right)^{2}}
\end{gathered}
$$

leads to the free energy minimum given by

$$
(\Delta F)_{\min }=-\frac{1}{2} \frac{\alpha^{2}}{\beta}
$$


One can see from Eqs. (17) and (20) that when $\alpha=0$ for a state with $\langle e\rangle=0$ then its value is less than zero for a nonzero $\langle e\rangle$. Therefore according to Eq. (22) even an infinitesimal impurity scattering rate stabilizes states with $\langle e\rangle \neq 0$ over $\langle e\rangle=0$ states. In other words the critical temperature in the presence of nonmagnetic impurities given by 23,24

$$
\ln \left(\frac{T_{c}}{T_{c_{0}}}\right)=\left(\langle e\rangle^{2}-1\right)\left(\psi\left(\frac{1}{2}+\varrho_{c}\right)-\psi\left(\frac{1}{2}\right)\right)
$$

with $\varrho_{c}$ as the value of $\varrho$ at $T_{c}$, is higher for the states with a nonzero $\langle e\rangle$ value than those characterized by $\langle e\rangle=0$. It is also worth mentioning that the states characterized by the same value of $\langle e\rangle$ are degenerate. We show the solutions of Eq. (23) for the states from Tab. 2 in the case of a not broken tetragonal symmetry $\left(c_{x} / c_{y}=1\right)$ in Fig. 2 , and for an orthorhombic symmetry with $c_{x} / c_{y}=0.8$ in Fig. 3. Comparison of Fig. 1a $\left(\right.$ at $\left.c_{x} / c_{y}=1\right)$ and Fig. 2 shows a degeneracy of the states with the same $\langle e\rangle$ value. When the FS symmetry is lowered to $C_{2 v}$ the states fall into two irreducible representations $\Gamma_{1}^{+}$and $\Gamma_{3}^{+}$(Tab. 1). The two $\Gamma_{3}^{+}$states (curves 3 and 6 in Fig. 3) are still degenerate as $\langle e\rangle=0$ in that case

(Fig. 1a). On the other hand most of $\Gamma_{1}^{+}$states are influenced by the orthorhombicity of the system and their critical temperatures are split. We present the evaluation of $T_{c}$ for the states under consideration at the impurity scattering level25 $\varrho_{c} T_{c} / T_{c_{0}}=0.1$ as a function of orthorhombic anisotropy parameter $c_{x} / c_{y}$ in Fig. 4. As mentioned earlier, the critical temperature depends on the order parameter and FS symmetries through $\langle e\rangle$ only. This leads to a remarkable similarity between the effect of FS orthorhombicity on $T_{c}$ (Fig. 4) and on $\langle e\rangle$ (Fig. 1a).

\section{SPECIFIC HEAT JUMP AT THE PHASE TRANSITION}

The specific heat jump at $T_{c}, \Delta C\left(T_{c}\right)$, normalized by the normal state specific heat $C_{N}\left(T_{c}\right)$ is given by

$$
\frac{\Delta C\left(T_{c}\right)}{C_{N}\left(T_{c}\right)}=\frac{12}{\left(f_{1}\right)_{T=T_{c}}}\left[1+T_{c}\left(\frac{d f_{0}}{d T}\right)_{T=T_{c}}\right]^{2}
$$

and $f_{0}$ from Eq. (17) yields 


$$
\frac{\Delta C\left(T_{c}\right)}{C_{N}\left(T_{c}\right)}=\frac{12}{f_{1}\left(\varrho_{c}\right)}\left[1+\left(\langle e\rangle^{2}-1\right) \varrho_{c} \psi^{(1)}\left(\frac{1}{2}+\varrho_{c}\right)\right]^{2}
$$

where $\varrho_{c}$ is $\varrho$ at $T=T_{c}$. This rather cumbersome formula, when considered along with Eq. (18), reduces significantly for $\langle e\rangle=0$ case

$$
\frac{\Delta C\left(T_{c}\right)}{C_{N}\left(T_{c}\right)}=\frac{12\left[1-\varrho_{c} \psi^{(1)}\left(\frac{1}{2}+\varrho_{c}\right)\right]^{2}}{\frac{\mu}{6} \varrho^{(3)}\left(\frac{1}{2}+\varrho_{c}\right)-\frac{1}{2}\left\langle e^{4}\right\rangle \psi^{(2)}\left(\frac{1}{2}+\varrho_{c}\right)}
$$

where $\mu=\left(1-c^{2}\right) /\left(1+c^{2}\right)$. For an appropriate choice of $\left\langle e^{4}\right\rangle$ value $\Delta C\left(T_{c}\right) / C_{N}\left(T_{c}\right)$ from Eq. (26) agrees with the result obtained by Hirschfeld et al. 17 as well as with that obtained by Suzumura and Schulz 26 in the Born limit. For a pure system 13 where $\varrho_{c}=0$

$$
\left(\frac{\Delta C\left(T_{c}\right)}{C_{N}\left(T_{c}\right)}\right)_{\varrho_{c}=0}=-\frac{24}{\psi^{(2)}\left(\frac{1}{2}\right)\left\langle e^{4}\right\rangle} \approx \frac{1.426}{\left\langle e^{4}\right\rangle}
$$

Alternatively, in a highly impure superconductor with $\varrho_{c} \rightarrow \infty$ i.e. $T_{c} \rightarrow 0$ due to suppression by the impurities, the two cases, depending on $\langle e\rangle$ value, are to be distinguished 13 First, when $\langle e\rangle \neq 0$ leads to

$$
\left(\frac{\Delta C\left(T_{c}\right)}{C_{N}\left(T_{c}\right)}\right)_{\varrho_{c} \rightarrow \infty}=-\frac{24}{\psi^{(2)}\left(\frac{1}{2}\right)} \approx 1.426
$$

and the second, with $\langle e\rangle=0$ yields

$$
\left(\frac{\Delta C\left(T_{c}\right)}{C_{N}\left(T_{c}\right)}\right)_{\varrho_{c} \rightarrow \infty}=0
$$

We note, that the specific heat jump value in $\varrho_{c} \rightarrow \infty$ limit for a nonzero value of $\langle e\rangle$ given by Eq. (28) agrees with that of an isotropic $s$-wave superconductor. This fact has a simple intuitive interpretation. A nonzero Fermi surface average of the order parameter leads to an asymptotic power-law critical temperature suppression for large impurity concentration 15 $T_{c} \sim\left(T_{c_{0}}\right)^{1 /\langle e\rangle^{2}}\left[\Gamma /\left(c^{2}+1\right)\right]^{\left(1-1 /\langle e\rangle^{2}\right)}$, therefore $T_{c}$ is almost constant for large $\Gamma$ values. The impurity effect, then, in the high impurity concentration range is the same as in the case of $s$-wave superconductivity, where $T_{c}$ is not changed by the nonmagnetic impurities. Alternatively, for $\langle e\rangle=0$ we observe a strong impurity-induced suppression of the critical 
temperature 23, 24 leading to its zero value at finite impurity concentration, which is reflected by a zero specific heat jump limit value in Eq. (29). Therefore the jump in the specific heat at $T_{c} \rightarrow 0$ approaches a value of 1.426 for the states belonging to $\Gamma_{1}^{+}$representation if their $\langle e\rangle$ is nonzero, whereas it decreases to zero for all the other states described by the non identity irreducible representations for which $\langle e\rangle=0$. Indeed, as it has been shown for the representative order parameters, 27.28 the gap anisotropy is smeared out by the isotropic impurity scattering in the first case $(\langle e\rangle \neq 0)$ and the density of states approaches that of an isotropic $s$-wave superconductor. The nonmagnetic impurities, however, are severe pairbreakers in a state with $\langle e\rangle=0$ and lead to a finite density of states at the Fermi energy. This qualitative difference is expected to be reflected in the quantities proportional to the density of states, for instance in the specific heat. According to the classification given in Tab. 2, in the limit of $T_{c} \rightarrow 0$ one should observe the BCS specific heat jump value for a $d_{x^{2}-y^{2}}$ state in YBCO $\left(\Gamma_{1}^{+}\right.$representation), but the jump should vanish in BSCCO $\left(\Gamma_{3}^{+}\right.$ representation). On the other hand $d_{x y}$ state in YBCO should lead to a zero jump in $T_{c} \rightarrow 0$ limit, but the BCS-like jump in BSCCO. In the extended $s$-wave state $k_{x} k_{y}\left(k_{x}^{2}-k_{y}^{2}\right)$, which belongs to $\Gamma_{3}^{+}$representation in both YBCO and BSCCO structures, the specific heat jump should decrease to zero with $T_{c} \rightarrow 0$ in both compounds. The results for specific heat jump in Born and unitary scattering limits are shown in Figs. 5 and 6 respectively for a circular Fermi surface $\left(c_{x} / c_{y}=1\right)$. The YBCO-type FS with a small orthorhombic anisotropy $\left(c_{x} / c_{y}=0.8\right)$ leads to the solutions presented in Fig. 7 (Born scattering) and Fig. 8 (unitary scattering). Finally, Figs. 9 and 10 correspond to both considered impurity scattering limits in a system with a Fermi surface of a large orthorhombicity given by $c_{x} / c_{y}=0.2$. In the case of a not broken tetragonal symmetry (Figs. 5 and 6) the specific heat jump of the identity representations $\Gamma_{1}^{+}$with $\langle e\rangle \neq 0$ (curves $\left.1,4,5\right)$, is almost constant and nonzero, but that of the other $\Gamma_{2}^{+}, \Gamma_{3}^{+}$and $\Gamma_{4}^{+}$irreducible representations with $\langle e\rangle=0$ (curves 6,2 and 3 respectively) is suppressed to zero by the impurities. It is also remarkable in Figs. 5 and 6 that the states having the same values of $\langle e\rangle,\left\langle e^{3}\right\rangle$ and $\left\langle e^{4}\right\rangle$ averages (compare Figs. 1a-1c at $c_{x} / c_{y}=1$ ) display the same values of the jump in the specific heat. When the 
FS symmetry changes into the orthorhombic there are four $\Gamma_{1}^{+}$representations in the set of considered functions (Tabs. 1, 2). We look at YBCO symmetry first. Except for two states of $\Gamma_{3}^{+}$symmetry $\left(k_{x} k_{y}, k_{x} k_{y}\left(k_{x}^{2}-k_{y}^{2}\right)\right)$ which are strongly suppressed by the nonmagnetic impurity scattering, the specific heat jump of all the other states goes to a BCS value of 1.426 in $T_{c} \rightarrow 0$ limit. For a small orthorhombic anisotropy a dramatic rise of the specific heat jump is seen only in the $d_{x^{2}-y^{2}}$ state (Figs. 7 and 8), whereas for a large FS anisotropy this increase, however not so abrupt, is observed for all four identity representations (Figs. 9 and 10). In the case of BSCCO symmetry (see Tab. 2) the sharp rise in the specific heat jump for a small orthorhombic FS anisotropy (Figs. 7 and 8) will be observed only for the $d_{x y}$ state. The $d_{x^{2}-y^{2}}$ state will not mix with the identity representations in BSCCO, which will lead to a zero value of the specific heat jump in the large impurity scattering limit. Therefore observation of a sharp rise in the specific heat jump at the phase transition with the transition temperature $T_{c} \rightarrow 0$ in dirty BSCCO would provide information about the possible presence of $d_{x y}$ state in the condensate. Note that in impure YBCO the same observation would signal a realization of the $d_{x^{2}-y^{2}}$ state. We do not consider the mixed states since it would require an analysis of many cases even if we confine ourselves to the representations from Tab. 1. However, one should take into account that for a small FS anisotropy (Figs. 7 and 8 ) the rise in the specific heat jump at $T_{c} \rightarrow 0$ will be less pronounced for a superposition of some $\Gamma_{1}^{+}$states than for a single $d_{x^{2}-y^{2}}$ state in the case of YBCO or a $d_{x y}$ one in BSCCO. Comparing the effect of Born scattering on the jump in the specific heat (Figs. 5, 7, 9) with that of the unitary impurity scattering (Figs. 6, 8, 10), we notice a difference in the range of medium impurity concentration for the states with the small $\langle e\rangle$ values (see Fig. 1a). It means that the states suppressed by the impurity scattering the most, display the largest difference between these two scattering limits, which is particularly seen in the states of $\langle e\rangle=0$, where the pair-breaking effect of the impurities is the strongest (Eq. (23)). 


\section{CONCLUSION}

The orthorhombic anisotropy of the cuprates results in a number of states belonging to the identity irreducible representation $\left(\Gamma_{1}^{+}\right)$. For these states the average value of the order parameter over the Fermi surface $(\langle e\rangle)$ may not vanish. The behavior for states with $\langle e\rangle \neq 0$ is qualitatively different than for states with $\langle e\rangle=0$. We have studied the effect of nonmagnetic impurity scattering on the superconducting states which may be realized

in the high temperature superconductors, distinguishing between the orthorhombicity of YBCO and BSCCO compounds. It has been pointed out, that the potential scattering stabilizes the $\Gamma_{1}^{+}$states (with $\langle e\rangle \neq 0$ ) against those with $\langle e\rangle=0$. A very interesting feature may be observed in the electronic specific heat jump at the phase transition for a small crystal orthorhombicity. The $d_{x^{2}-y^{2}}$ state in YBCO and the $d_{x y}$ state in BSCCO are predicted to lead to a sharp rise in the jump in specific heat at $T_{c}$ in the limit $T_{c} \rightarrow 0$ when the critical temperature is suppressed by the potential impurity scattering. This effect may be helpful in the identification of the superconducting states in the cuprates.

\section{ACKNOWLEDGMENT}

Work supported by the Natural Sciences and Engineering Research Council of Canada. 


\section{APPENDIX:}

In this appendix we present the Fermi surface averages of the 1-st, 3-rd and 4-th powers of the normalized order parameter function $e(\mathbf{k})$ for the states considered in the paper. The

average values were taken over the YBCO-type Fermi surface given by Eq. (2). To obtain the results for BSCCO one should use the equivalence between the states in YBCO and BSCCO geometry summarized in Tab. 2.

$$
\begin{aligned}
& e(\mathbf{k})=\left(k_{x}^{2} \pm k_{y}^{2}\right)\left\langle\left(k_{x}^{2} \pm k_{y}^{2}\right)^{2}\right\rangle^{-1 / 2} \\
& \nu\left(\frac{c_{x}}{c_{y}}\right)=\left[\frac{3}{2} \pm \frac{c_{x}}{c_{y}}+\frac{3}{2}\left(\frac{c_{x}}{c_{y}}\right)^{2}\right]^{-1 / 2} \\
& \langle e\rangle=\nu\left(\frac{c_{x}}{c_{y}}\right)\left[1 \pm \frac{c_{x}}{c_{y}}\right] \\
& \left\langle e^{3}\right\rangle=\nu^{3}\left(\frac{c_{x}}{c_{y}}\right)\left[\frac{5}{2}\left(1 \pm\left(\frac{c_{x}}{c_{y}}\right)^{3}\right)^{ \pm} \frac{3}{2} \frac{c_{x}}{c_{y}}\left(1 \pm \frac{c_{x}}{c_{y}}\right)\right] \\
& \left\langle e^{4}\right\rangle=16 \nu^{4}\left(\frac{c_{x}}{c_{y}}\right)\left[\frac{35}{128}\left(1 \mp \frac{c_{x}}{c_{y}}\right)^{4}-\frac{5}{4}\left(1 \mp \frac{c_{x}}{c_{y}}\right)^{3}+\frac{9}{4}\left(1 \mp \frac{c_{x}}{c_{y}}\right)^{2}-2\left(1 \mp \frac{c_{x}}{c_{y}}\right)+1\right. \\
& \left.\langle e\rangle=0,\left\langle e^{3}\right\rangle=0,\left\langle e^{4}\right\rangle=\frac{3}{2}\right] \\
& \frac{e(\mathbf{k})=\left(k_{x} k_{y}\right)\left\langle\left(k_{x} k_{y}\right)^{2}\right\rangle}{-1 / 2}
\end{aligned}
$$$$
\underline{e(\mathbf{k})=\left(k_{x}^{2} k_{y}^{2}\right)\left\langle\left(k_{x}^{2} k_{y}^{2}\right)^{2}\right\rangle^{-1 / 2}}
$$

$$
\langle e\rangle=\left(\frac{2}{3}\right)^{1 / 2}, \quad\left\langle e^{3}\right\rangle=\frac{5}{3}\left(\frac{2}{3}\right)^{1 / 2}, \quad\left\langle e^{4}\right\rangle=\frac{35}{18}
$$




$$
\underline{e(\mathbf{k})=\left(k_{x} k_{y}\left(k_{x}^{2}-k_{y}^{2}\right)\right)\left\langle\left(k_{x} k_{y}\left(k_{x}^{2}-k_{y}^{2}\right)\right)^{2}\right\rangle^{-1 / 2}}
$$

$$
\langle e\rangle=0, \quad\left\langle e^{3}\right\rangle=0
$$

$$
\left\langle e^{4}\right\rangle=\left[\frac{5}{128}\left(1+\left(\frac{c_{x}}{c_{y}}\right)^{2}\right)-\frac{3}{64} \frac{c_{x}}{c_{y}}\right]^{-2}\left[d_{1}\left(1+\left(\frac{c_{x}}{c_{y}}\right)^{4}\right)-4 d_{2} \frac{c_{x}}{c_{y}}\left(1+\left(\frac{c_{x}}{c_{y}}\right)^{2}\right)+6 d_{3}\left(\frac{c_{x}}{c_{y}}\right)^{2}\right]
$$

$$
\underline{e(\mathbf{k})=\left(k_{x}^{2}-k_{y}^{2}\right)^{2}\left\langle\left(k_{x}^{2}-k_{y}^{2}\right)^{4}\right\rangle^{-1 / 2}}
$$

$$
\nu\left(\frac{c_{x}}{c_{y}}\right)=\left[\frac{35}{128}\left(1+\frac{c_{x}}{c_{y}}\right)^{4}-\frac{5}{4}\left(1+\frac{c_{x}}{c_{y}}\right)^{3}+\frac{9}{4}\left(1+\frac{c_{x}}{c_{y}}\right)^{2}-2\left(1+\frac{c_{x}}{c_{y}}\right)+1\right]^{-1 / 2}
$$$$
\langle e\rangle=\nu\left(\frac{c_{x}}{c_{y}}\right) \frac{1}{4}\left(\frac{3}{2}-\frac{c_{x}}{c_{y}}+\frac{3}{2}\left(\frac{c_{x}}{c_{y}}\right)^{2}\right)
$$$$
\left\langle e^{3}\right\rangle=\nu^{3}\left(\frac{c_{x}}{c_{y}}\right)\left[d_{6}\left(1+\left(\frac{c_{x}}{c_{y}}\right)^{6}\right)-6 d_{7} \frac{c_{x}}{c_{y}}\left(1+\left(\frac{c_{x}}{c_{y}}\right)^{4}\right)+15 d_{8}\left(\frac{c_{x}}{c_{y}}\right)^{2}\left(1+\left(\frac{c_{x}}{c_{y}}\right)^{2}\right)\right.
$$$$
\left.-20 d_{9}\left(\frac{c_{x}}{c_{y}}\right)^{3}\right]
$$

$$
\begin{aligned}
& \left\langle e^{4}\right\rangle=\nu^{4}\left(\frac{c_{x}}{c_{y}}\right)\left[d_{4}\left(1+\left(\frac{c_{x}}{c_{y}}\right)^{8}\right)-8 d_{5} \frac{c_{x}}{c_{y}}\left(1+\left(\frac{c_{x}}{c_{y}}\right)^{6}\right)+28 d_{1}\left(\frac{c_{x}}{c_{y}}\right)^{2}\left(1+\left(\frac{c_{x}}{c_{y}}\right)^{4}\right)\right. \\
& \left.-56 d_{2}\left(\frac{c_{x}}{c_{y}}\right)^{3}\left(1+\left(\frac{c_{x}}{c_{y}}\right)^{2}\right)+70 d_{3}\left(\frac{c_{x}}{c_{y}}\right)^{4}\right]
\end{aligned}
$$

where 


$$
\begin{aligned}
& d_{1}=\frac{99}{32768}, d_{2}=\frac{45}{32768}, d_{3}=\frac{35}{32768}, d_{4}=\frac{6435}{32768}, d_{5}=\frac{429}{32768} \\
& d_{6}=\frac{231}{1024}, \quad d_{7}=\frac{21}{1024}, \quad d_{8}=\frac{7}{1024}, \quad d_{9}=\frac{5}{1024} .
\end{aligned}
$$




\section{REFERENCES}

* on leave from: Institute of Physics, Politechnika Wrocławska, Wybrzeże Wyspiańskiego 27, 50-370 Wrocław, Poland

${ }^{1}$ S. A. Sunshine et al., Phys. Rev. B38, 893 (1988); P. Bordet et al., Physica C 156, 189 (1988); V. Petricek et al., Phys. Rev. B42, 387 (1990)

${ }^{2}$ D. N. Basov, R. Liang, D. A. Bonn, W. N. Hardy, B. Dabrowski, M. Quijada, D. B. Tanner, J. P. Rice, D. M. Ginsberg and T. Timusk, Phys. Rev. Lett. 74, 598 (1995)

${ }^{3}$ K. Zhang, D. A. Bonn, S. Kamal, R. Liang, D. J. Baar, W. N. Hardy, D. N. Basov and T. Timusk, Phys. Rev. Lett. 73, 2484 (1994)

${ }^{4}$ J. L. Cohn, E. F. Skelton, S. A. Wolf, J. Z. Liu and R. N. Shelton, Phys. Rev. B45, 13144 $(1992)$

${ }^{5}$ R. C. Yu, M. B. Salamon, J. P. Lu and W. C. Lee, Phys. Rev. Lett. 69, 1431 (1992)

${ }^{6}$ Z. Schlesinger, R. T. Collins, L. D. Rotter, F. Holtzberg, C. Feild, U. Welp, G. W. Crabtree, J. Z. Liu, Y. Fang, K. G. Vandervoort and S. Fleshler, Physica C 235-240, 49 (1994)

${ }^{7}$ T. A. Friedmann, M. W. Rabin, J. Giapintzakis, J. P. Rice and D. M. Ginsberg, Phys. Rev. B42, 6217 (1990)

${ }^{8}$ R. Gagnon, C. Lupien and L. Taillefer, Phys. Rev. B50, 3458 (1994)

${ }^{9}$ M. A. Quijada, D. B. Tanner, R. J. Kelley and M. Onellion, Physica C 235-240, 1123 $(1994)$

${ }^{10}$ P. B. Allen, W. E. Pickett and H. Krakauer, Phys. Rev. B37, 7482 (1988)

${ }^{11}$ C. O’Donovan and J. P. Carbotte, Phys. Rev. B52, 4568 (1995)

12 J. Annett, N. Goldenfeld and A. J. Leggett, in Physical Properties of High Temperature 
Superconductors, Vol. 5, D. M. Ginsberg (ed.), (World Scientific, Singapore, 1996)

${ }^{13}$ G. Harań, J. Taylor and A. D. S. Nagi, preprint

${ }^{14}$ G.F. Koster et al, Properties of the thirty-two point groups (M.I.T. Press, Cambridge, 1963)

${ }^{15}$ H. Kim and E. J. Nicol, Phys. Rev. B52, 13576 (1995)

${ }^{16}$ P. J. Hirschfeld, D. Vollhardt and P. Wölfle, Solid State Commun. 59, 111 (1986); J. Keller, K. Scharnberg and H. Monien, Physica C 152, 302 (1988); C. J. Pethick and D. Pines, Phys. Rev. Lett. 57, 118 (1986)

${ }^{17}$ P. J. Hirschfeld, P. Wölfle and D. Einzel, Phys. Rev. B37, 83 (1988)

${ }^{18}$ The role of momentum-dependent impurity potential in anisotropic superconductors is discussed in G. Harań and A. D. S. Nagi, Phys. Rev. B54 (1996)

${ }^{19}$ K. Maki, in Superconductivity, R. D. Parks (ed.), (Marcel Dekker, New York, 1969), Vol. 2, pp. $1035-1102$

${ }^{20}$ P. J. Davis, in Handbook of Mathematical Functions, M. Abramowitz and I. A. Stegun (eds.) (Dover, New York, 1965) pp 253-266

${ }^{21}$ Since the normalization $\left\langle e^{2}\right\rangle=1$ we do not consider the $\left\langle e^{2}\right\rangle$ average. One can relax that constraint by replacing $e(\mathbf{k})$ with $e(\mathbf{k}) /\left\langle e^{2}\right\rangle^{1 / 2}$ in Eqs. (17) and (18).

${ }^{22}$ In the BCS theory $T_{c_{0}}=\left(2 \omega_{D} e^{\gamma} / \pi\right) \exp \left\{-1 /\left(N_{0} V_{0}\left\langle e^{2}\right\rangle\right)\right\}$, where $\omega_{D}$ is a cut-off energy and $\gamma$ is the Euler constant.

${ }^{23}$ A. A. Abrikosov, Physica C 214, 107 (1993)

${ }^{24}$ R. J. Radtke, K. Levin, H.-B. Schüttler and M. R. Norman, Phys. Rev. B48, 653 (1993)

${ }^{25}$ Note that the normalized scattering rate $\varrho_{c} T_{c} / T_{c_{0}}$ has a different meaning in the Born limit, where $\varrho_{c} T_{c} / T_{c_{0}}=\pi N_{0} n_{i} V_{i}^{2} /\left(2 \pi T_{c_{0}}\right)$ and in the unitary limit with $\varrho_{c} T_{c} / T_{c_{0}}=\Gamma /\left(2 \pi T_{c_{0}}\right)$. 
${ }^{26}$ Y. Suzumura and H. J. Schulz, Phys. Rev. B39, 11398 (1989)

${ }^{27}$ L. S. Borkowski and P. J. Hirschfeld, Phys. Rev. B49, 15404 (1994)

${ }^{28}$ R. Fehrenbacher and M. R. Norman, Phys. Rev. B50, 3495 (1994) 


\section{FIGURE CAPTIONS}

Fig. 1. The orthorhombic crystal field $c_{x} / c_{y}$ dependence of the Fermi surface averaged powers of the superconducting order parameter function $e(\mathbf{k})$ : a $\left.)\langle e\rangle, \mathrm{b})\left\langle e^{3}\right\rangle, \mathrm{c}\right)\left\langle e^{4}\right\rangle$. The figure labels correspond to the state numbers in Tab. 2.

Fig. 2. Normalized critical temperature $T_{c} / T_{c_{0}}$ of the states from Tab. 2 as a function of the normalized impurity scattering rate $\varrho_{c} T_{c} / T_{c_{0}}$ for the circular Fermi surface $\left(c_{x} / c_{y}=1\right)$.

Fig. 3. Normalized critical temperature $T_{c} / T_{c_{0}}$ of the states from Tab. 2 as a function of the normalized impurity scattering rate $\varrho_{c} T_{c} / T_{c_{0}}$ for the elliptical Fermi surface $\left(c_{x} / c_{y}=0.8\right)$.

Fig. 4. Normalized critical temperature $T_{c} / T_{c_{0}}$ of the states from Tab. 2 as a function of the Fermi surface orthorhombic anisotropy parameter $c_{x} / c_{y}$ for the impurity scattering rate $\varrho_{c} T_{c} / T_{c_{0}}=0.1$

Fig. 5. Jump in the specific heat at $T_{c}$ normalized by the normal specific heat at $T_{c}$ of the states from Tab. 2 as a function of the normalized impurity scattering rate $\varrho_{c} T_{c} / T_{c_{0}}$ in the Born limit for the circular Fermi surface $\left(c_{x} / c_{y}=1\right)$.

Fig. 6. Jump in the specific heat at $T_{c}$ normalized by the normal specific heat at $T_{c}$ of the states from Tab. 2 as a function of the normalized impurity scattering rate $\varrho_{c} T_{c} / T_{c_{0}}$ in the unitary limit for the circular Fermi surface $\left(c_{x} / c_{y}=1\right)$.

Fig. 7. Jump in the specific heat at $T_{c}$ normalized by the normal specific heat at $T_{c}$ of the states from Tab. 2 as a function of the normalized impurity scattering rate $\varrho_{c} T_{c} / T_{c_{0}}$ in the Born limit for the elliptical Fermi surface $\left(c_{x} / c_{y}=0.8\right)$. 
Fig. 8. Jump in the specific heat at $T_{c}$ normalized by the normal specific heat at $T_{c}$ of the states from Tab. 2 as a function of the normalized impurity scattering rate $\varrho_{c} T_{c} / T_{c_{0}}$ in the unitary limit for the elliptical Fermi surface $\left(c_{x} / c_{y}=0.8\right)$.

Fig. 9. Jump in the specific heat at $T_{c}$ normalized by the normal specific heat at $T_{c}$ of the states from Tab. 2 as a function of the normalized impurity scattering rate $\varrho_{c} T_{c} / T_{c_{0}}$ in the Born limit for the elliptical Fermi surface $\left(c_{x} / c_{y}=0.2\right)$.

Fig. 10. Jump in the specific heat at $T_{c}$ normalized by the normal specific heat at $T_{c}$ of the states from Tab. 2 as a function of the normalized impurity scattering rate $\varrho_{c} T_{c} / T_{c_{0}}$ in the unitary limit for the elliptical Fermi surface $\left(c_{x} / c_{y}=0.2\right)$. 


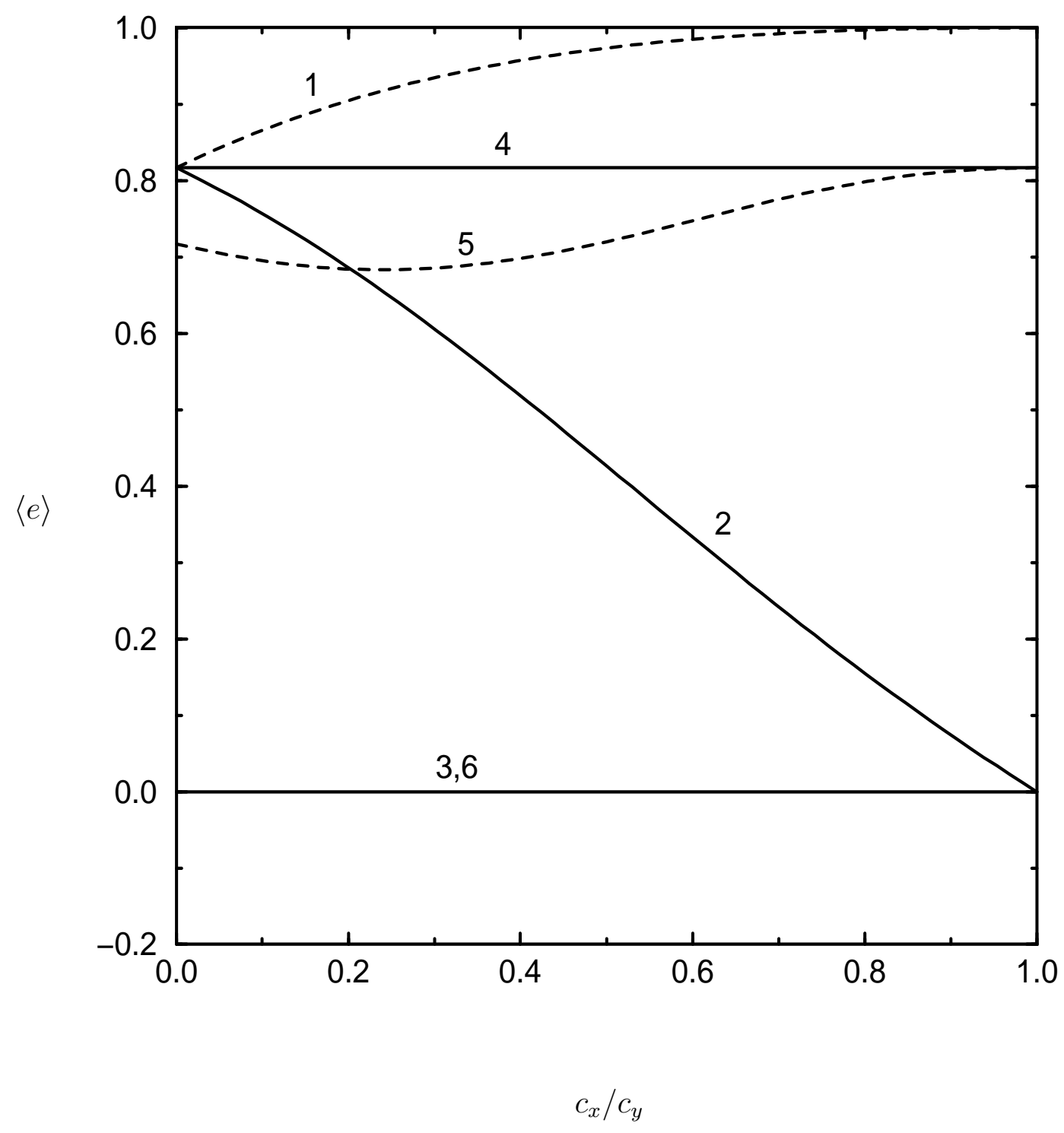




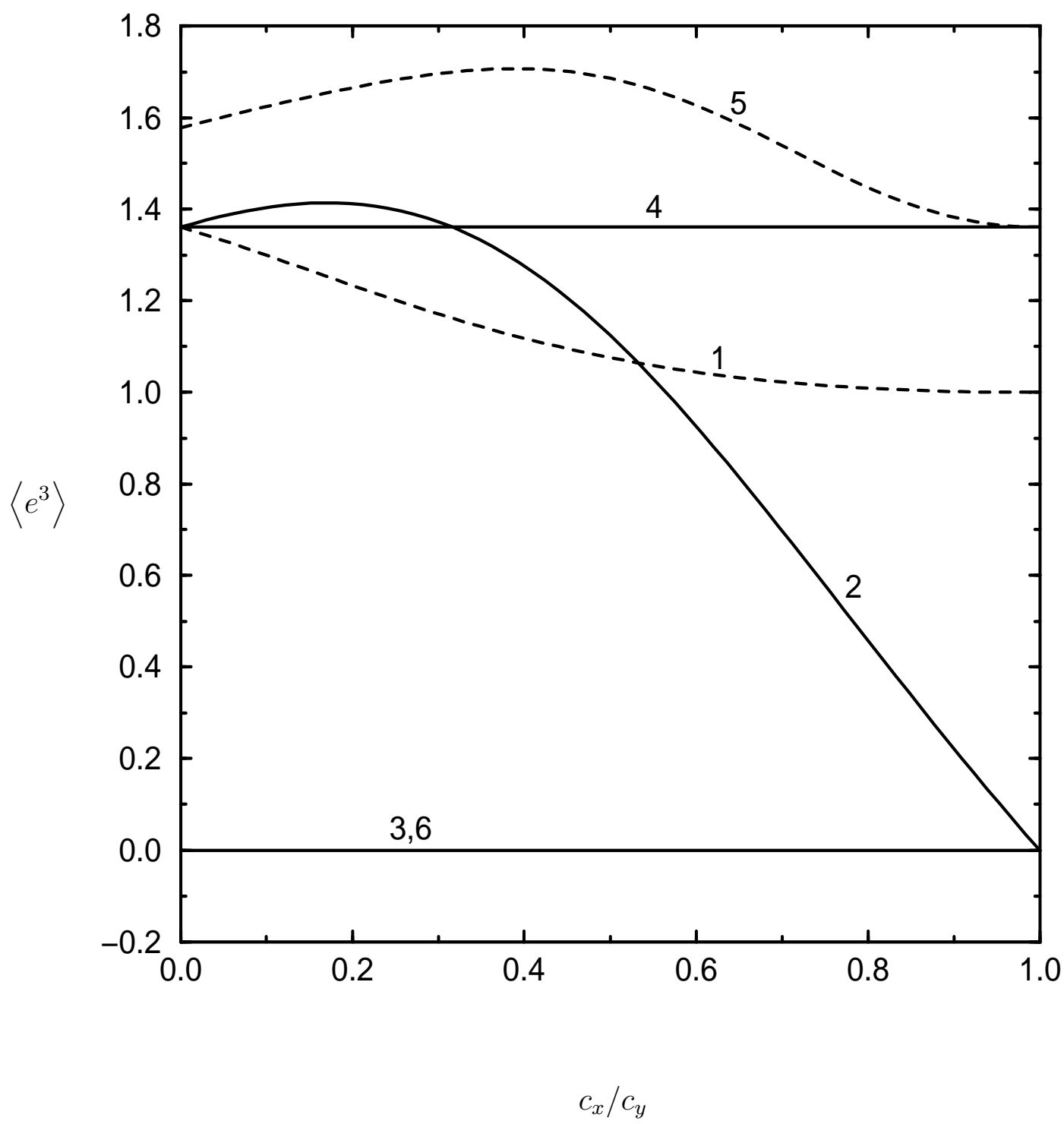




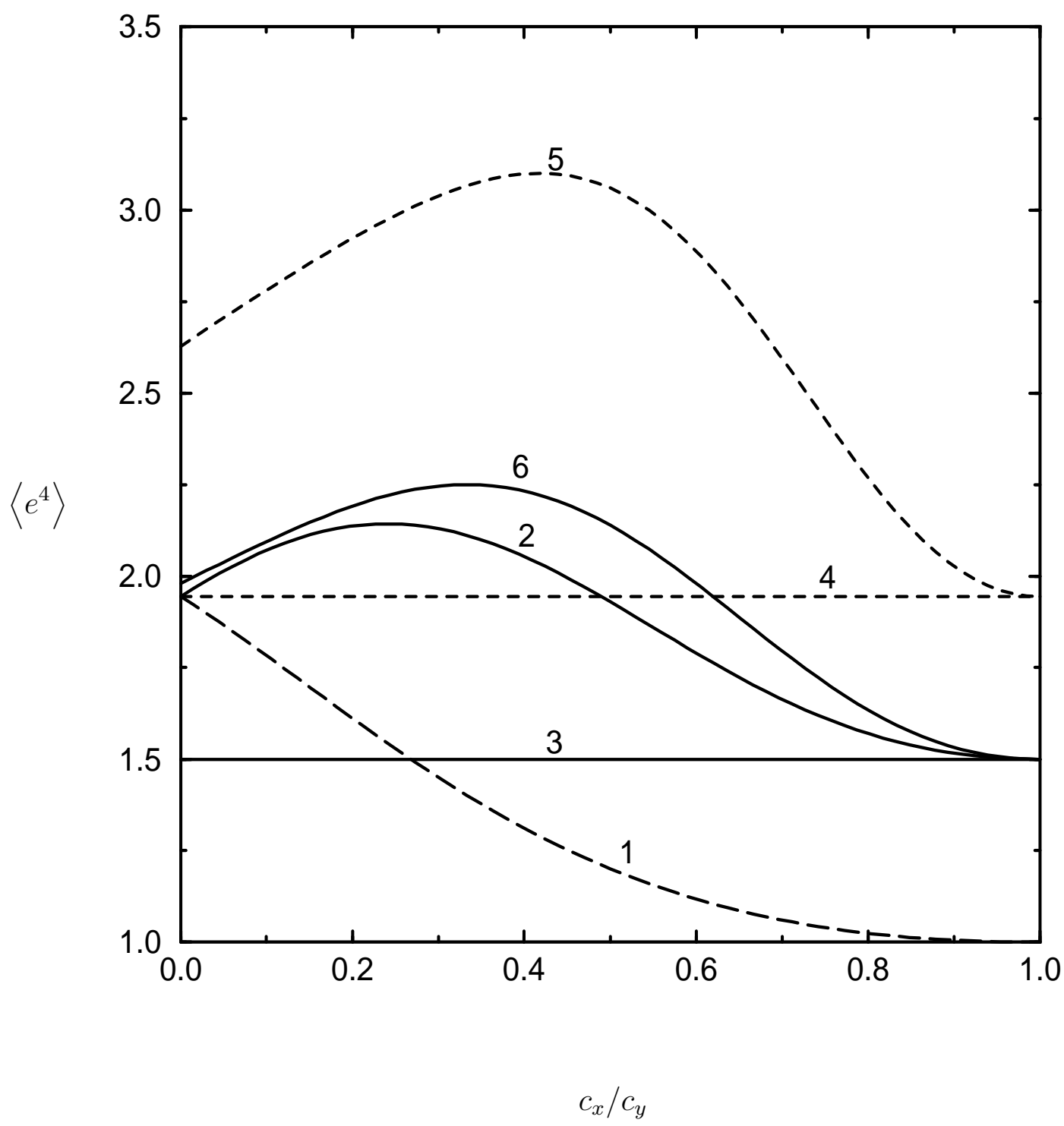




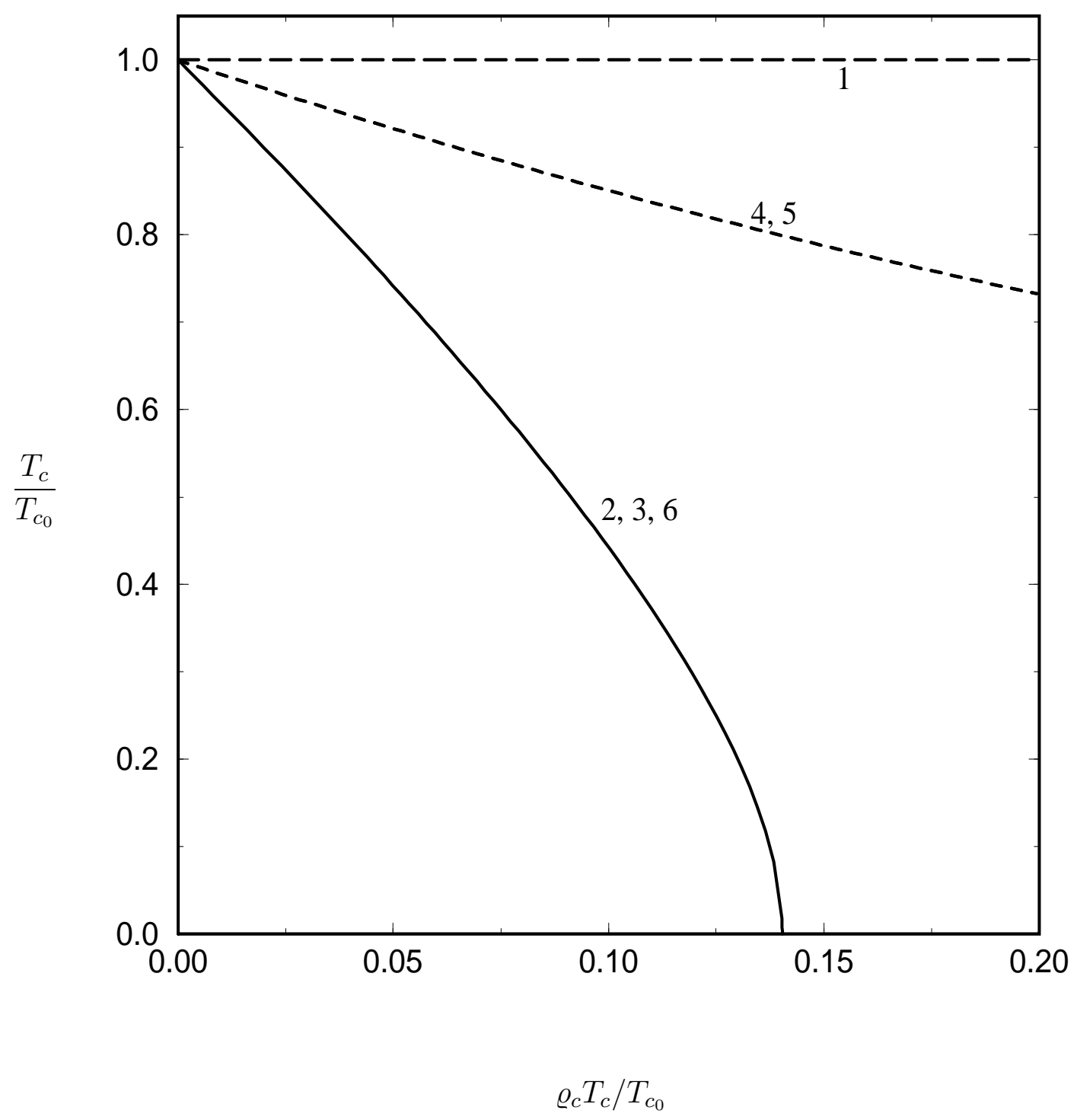




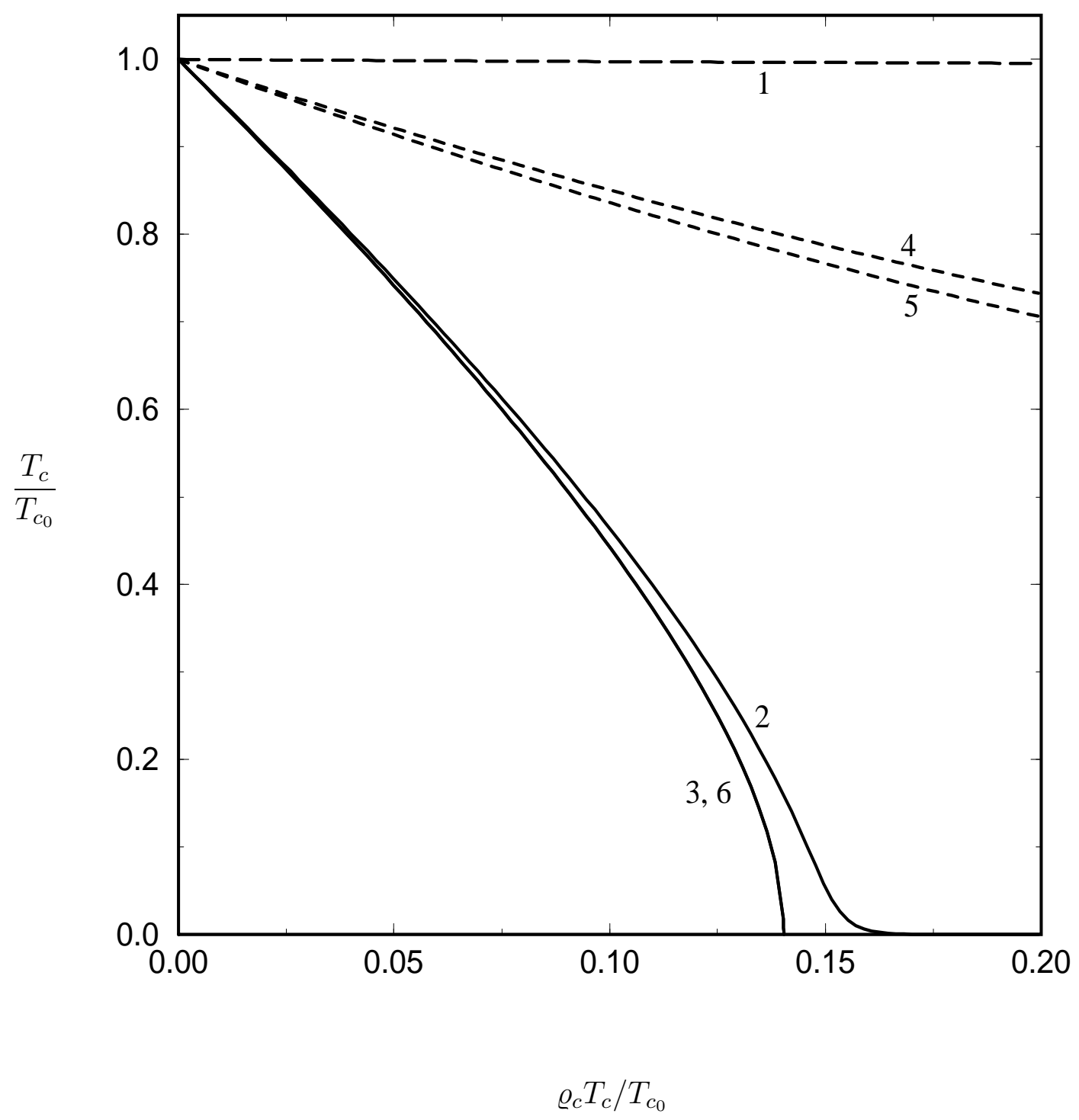




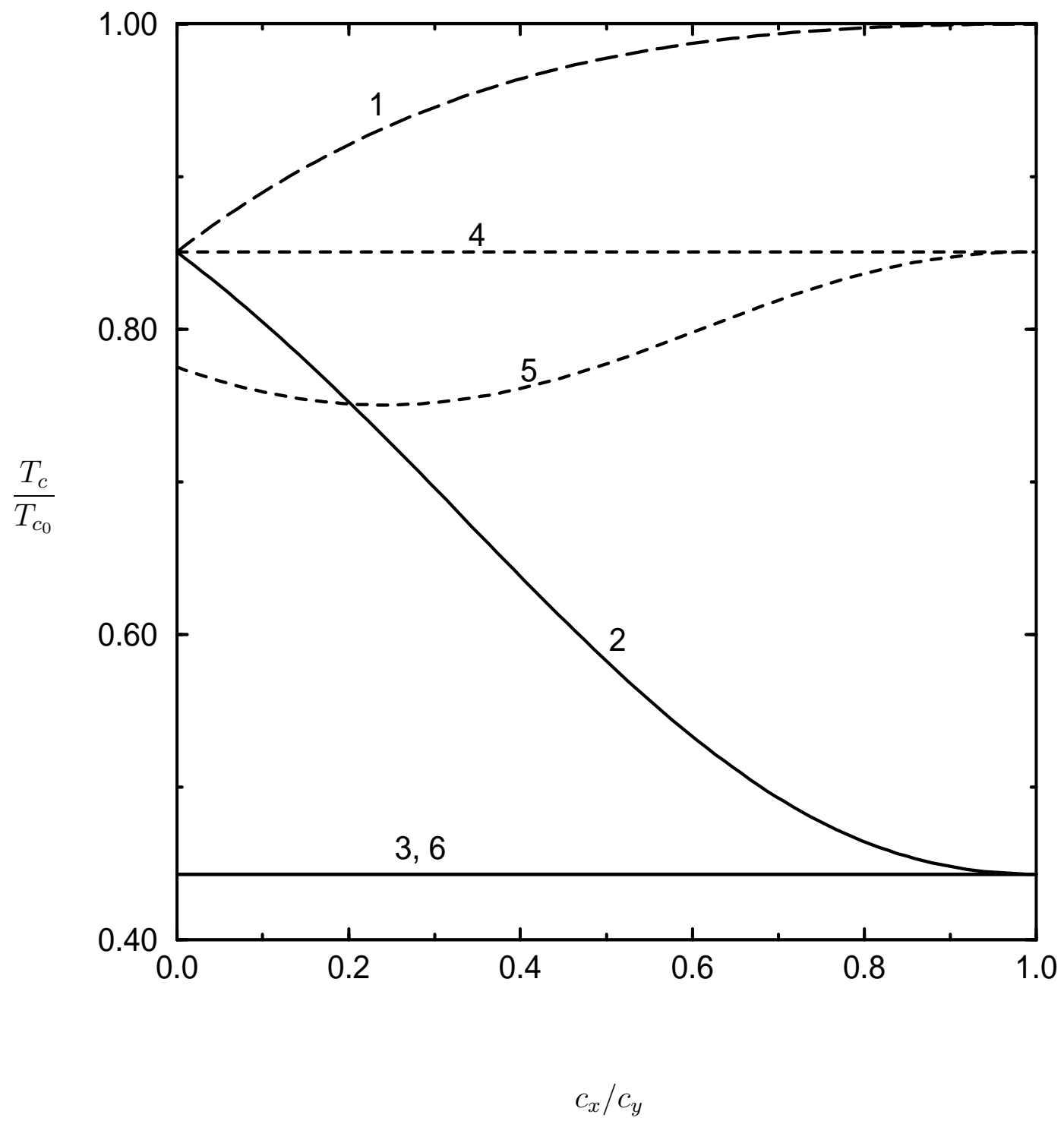




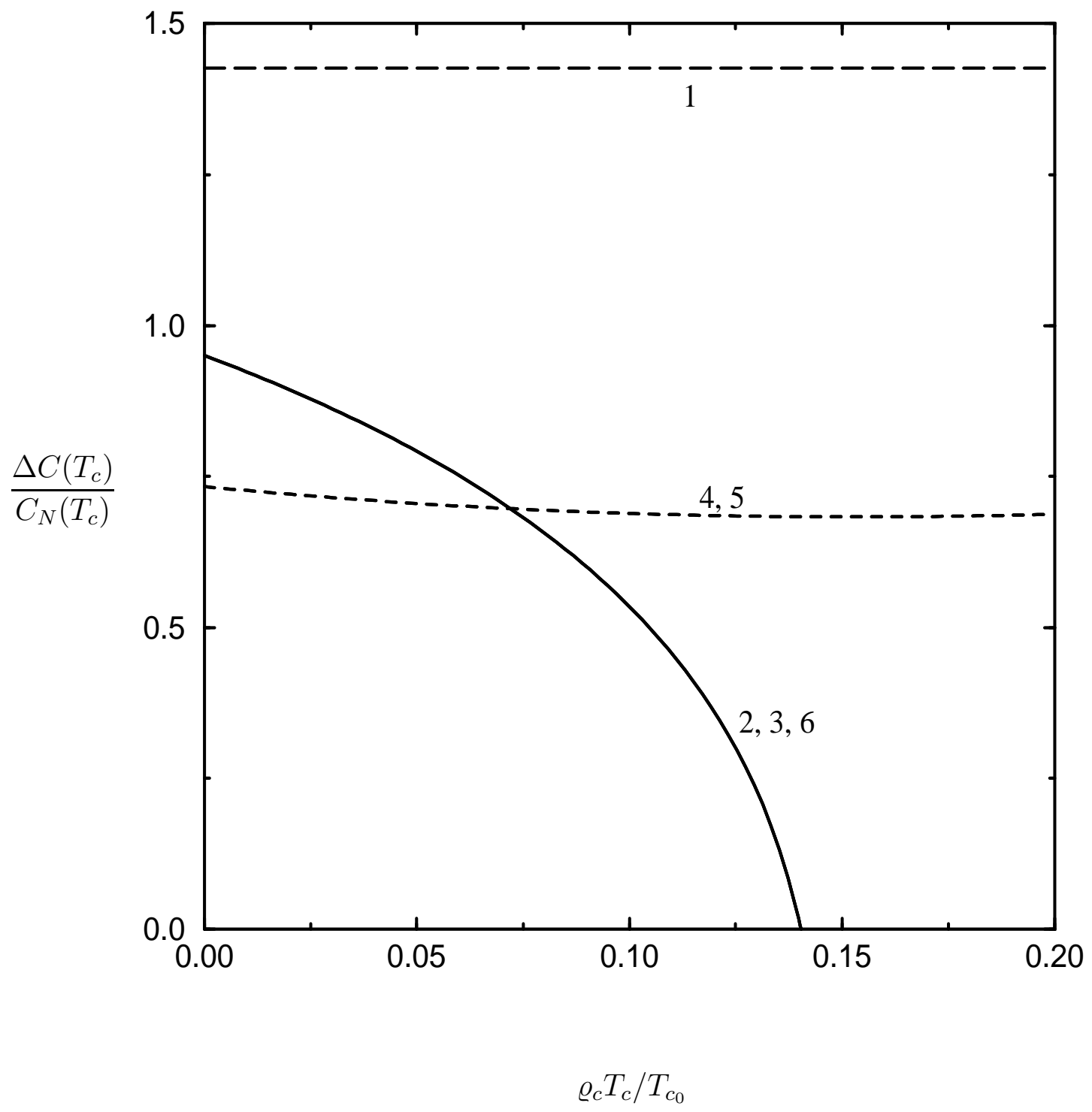




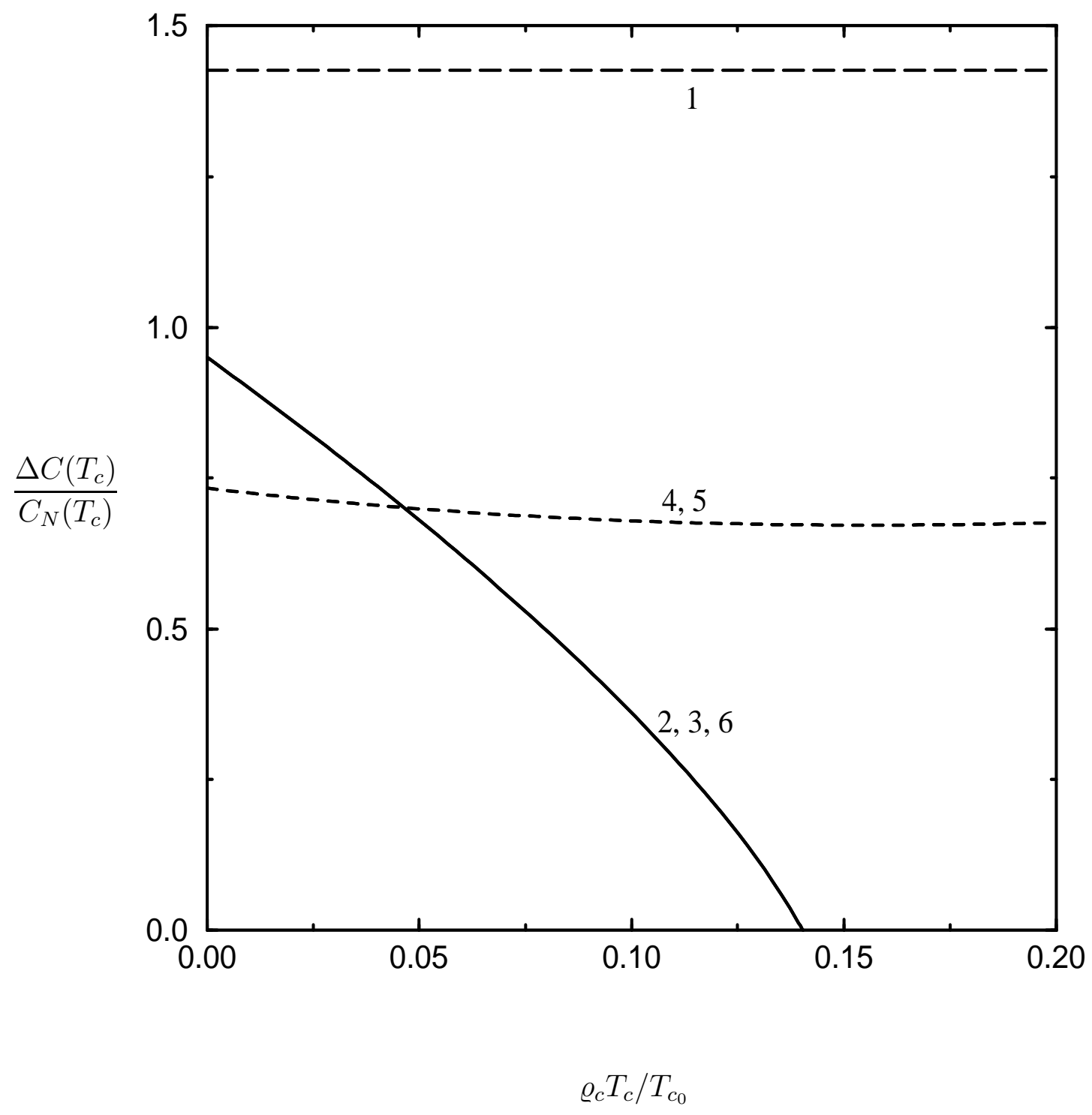




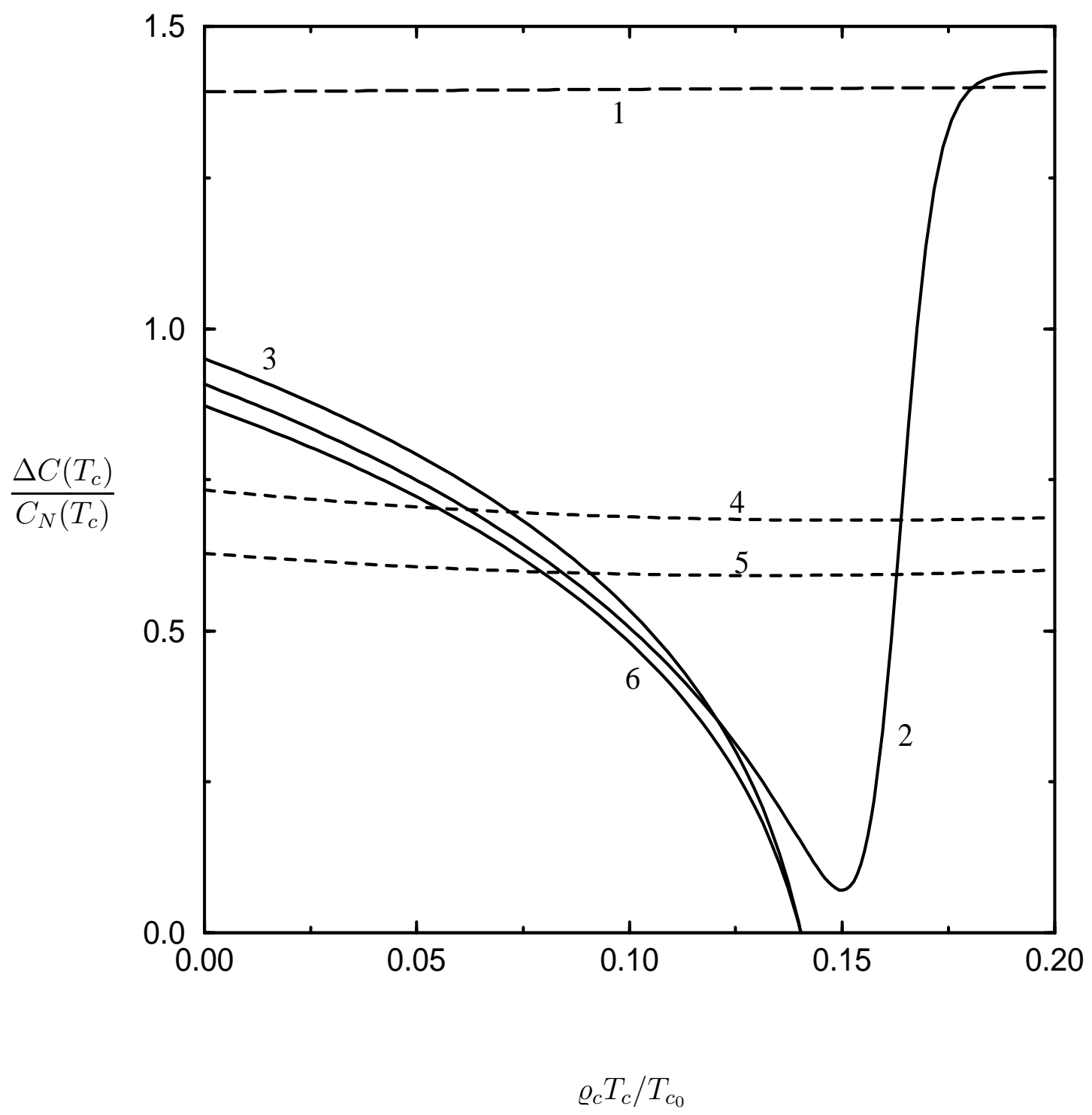




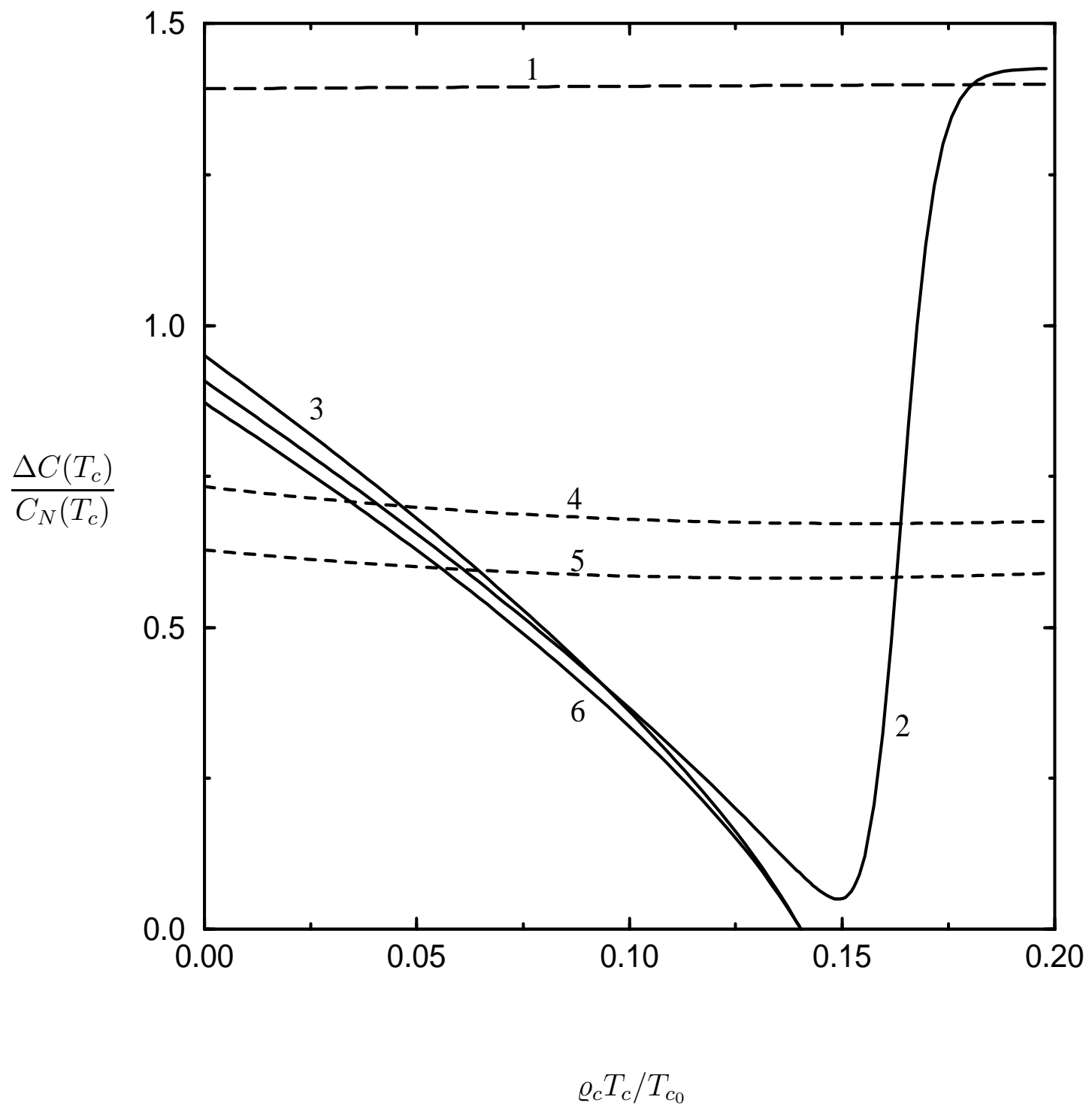




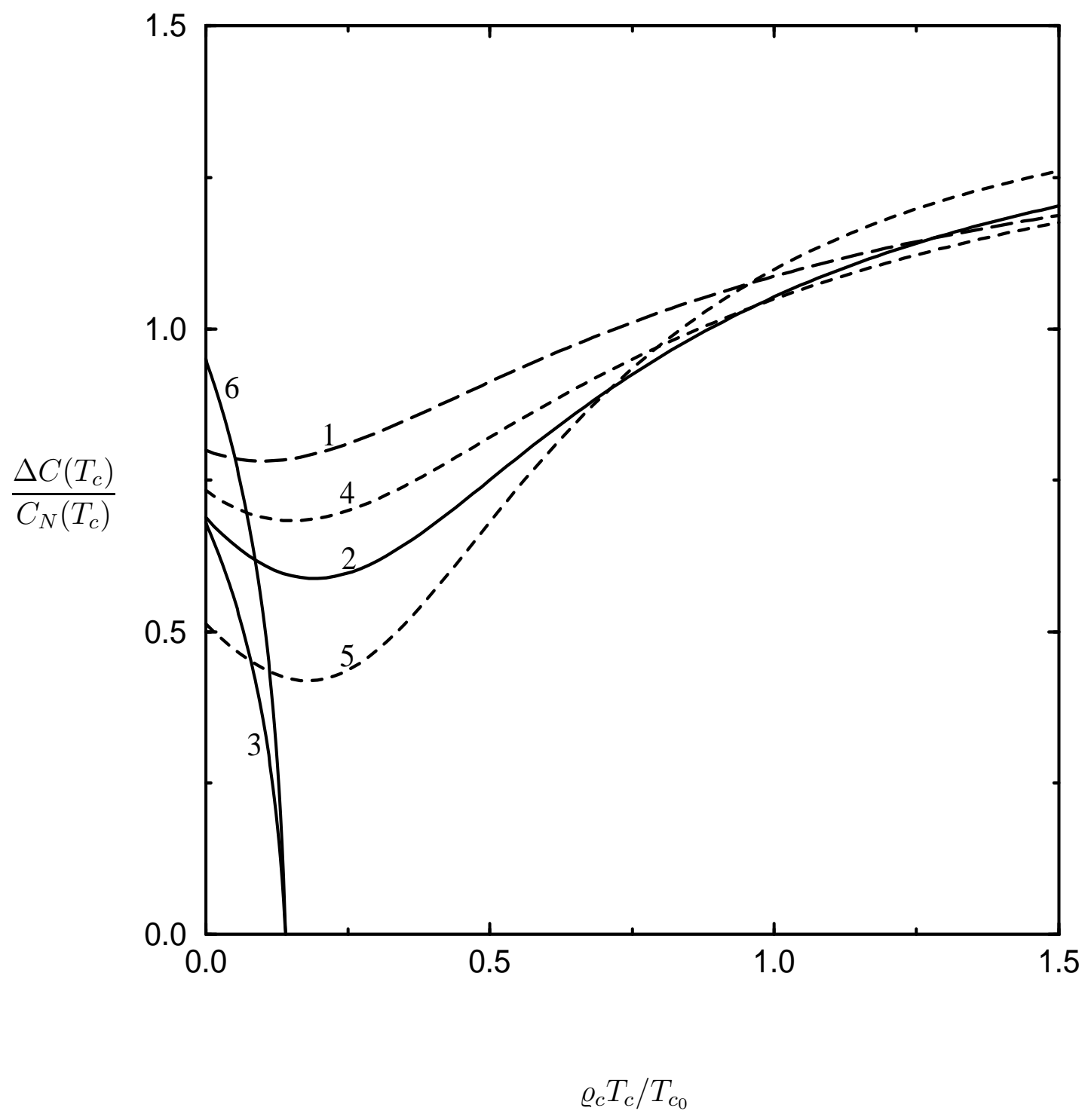




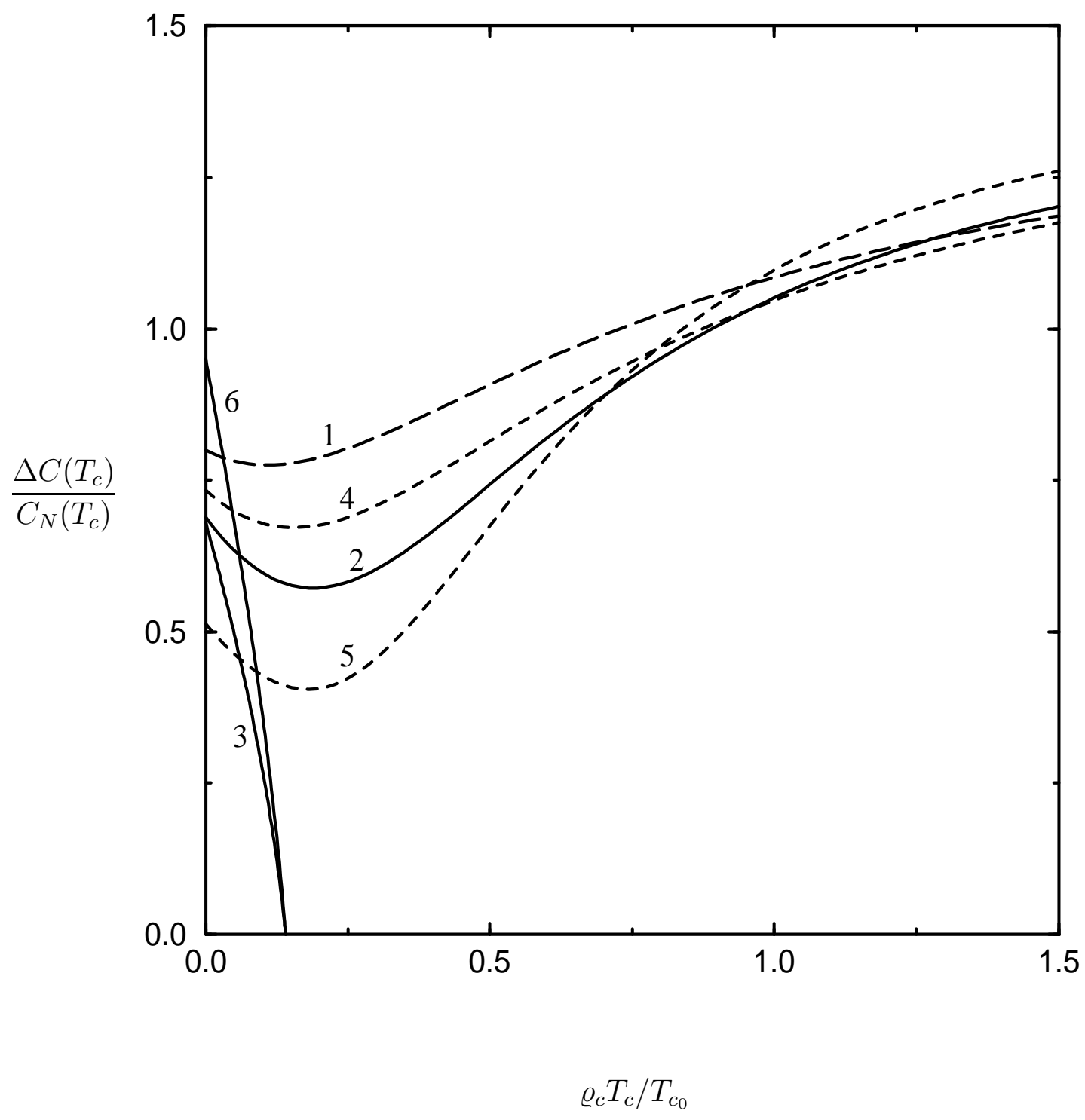


Table 1: Group classification of the superconducting order parameter $\Delta e(\mathbf{k})$.

\begin{tabular}{||c|c|c|c||}
\hline \hline \multicolumn{3}{||c||}{$\begin{array}{c}\text { Irreducible } \\
\text { representation } \Gamma\end{array}$} & \multirow{2}{*}{$\begin{array}{c}\text { basis function } \\
e(\mathbf{k})\end{array}$} \\
\cline { 2 - 3 }$C_{4 v}$ & \multicolumn{2}{|c|}{$C_{2 v}$} & \\
\cline { 2 - 3 } & $Y B C O$ & $B S C C O$ & \\
\hline$\Gamma_{1}^{+}$ & $\Gamma_{1}^{+}$ & $\Gamma_{1}^{+}$ & $k_{x}^{2}+k_{y}^{2}$ \\
$\Gamma_{3}^{+}$ & $\Gamma_{1}^{+}$ & $\Gamma_{3}^{+}$ & $k_{x}^{2}-k_{y}^{2}$ \\
$\Gamma_{4}^{+}$ & $\Gamma_{3}^{+}$ & $\Gamma_{1}^{+}$ & $k_{x} k_{y}$ \\
$\Gamma_{1}^{+}$ & $\Gamma_{1}^{+}$ & $\Gamma_{1}^{+}$ & $k_{x}^{2} k_{y}^{2}$ \\
$\Gamma_{1}^{+}$ & $\Gamma_{1}^{+}$ & $\Gamma_{1}^{+}$ & $\left(k_{x}^{2}-k_{y}^{2}\right)^{2}$ \\
$\Gamma_{2}^{+}$ & $\Gamma_{3}^{+}$ & $\Gamma_{3}^{+}$ & $k_{x} k_{y}\left(k_{x}^{2}-k_{y}^{2}\right)$ \\
\hline \hline
\end{tabular}


Table 2: $C_{2 v}$ irreducible representation basis functions in $\mathrm{YBCO}$ and $\mathrm{BSCCO}$ geometry.

\begin{tabular}{||c|c|c|c||}
\hline \hline No. & $\begin{array}{c}\text { Irreducible } \\
\text { representation }\end{array}$ & $Y B C O$ & $B S C C O$ \\
\hline 1 & $\Gamma_{1}^{+}$ & $k_{x}^{2}+k_{y}^{2}$ & $k_{x}^{2}+k_{y}^{2}$ \\
2 & $\Gamma_{1}^{+}$ & $k_{x}^{2}-k_{y}^{2}$ & $k_{x} k_{y}$ \\
3 & $\Gamma_{3}^{+}$ & $k_{x} k_{y}$ & $k_{x}^{2}-k_{y}^{2}$ \\
4 & $\Gamma_{1}^{+}$ & $k_{x}^{2} k_{y}^{2}$ & $\left(k_{x}^{2}-k_{y}^{2}\right)^{2}$ \\
5 & $\Gamma_{1}^{+}$ & $\left(k_{x}^{2}-k_{y}^{2}\right)^{2}$ & $k_{x}^{2} k_{y}^{2}$ \\
6 & $\Gamma_{3}^{+}$ & $k_{x} k_{y}\left(k_{x}^{2}-k_{y}^{2}\right)$ & $k_{x} k_{y}\left(k_{x}^{2}-k_{y}^{2}\right)$ \\
\hline \hline
\end{tabular}

